\title{
Nebulised budesonide using a novel device in patients with oral steroid- dependent asthma
}

\author{
Claus Vogelmeier ${ }^{1}$, Peter Kardos ${ }^{2}$, Thomas Hofmann ${ }^{3,4}$, Sebastian Canisius ${ }^{3}$, \\ Gerhard Scheuch ${ }^{3}$, Bernhard Muellinger ${ }^{3}$, Karlheinz Nocker ${ }^{3}$, Guenter Menz ${ }^{5}$ \\ and Klaus F. Rabe ${ }^{6}$
}

Affiliations: ${ }^{1}$ Dept of Medicine, Pulmonary and Critical Care Medicine, German Center for Lung Research (DZL), University Medical Center Giessen and Marburg, Philipps-University Marburg, Marburg, Germany. ${ }^{2}$ Respiratory Group Practice at Red Cross Maingau Hospital, Frankfurt, Germany. ${ }^{3}$ Vectura GmbH, Gemuenden, Germany. ${ }^{4}$ Aumapharma Inc., Doylestown, PA, USA. ${ }^{5}$ Hochgebirgsklinik Davos, Davos, Switzerland. ${ }^{6}$ LungenClinic Grosshansdorf, Grosshansdorf, Germany.

Correspondence: Klaus F. Rabe, LungenClinic Grosshansdorf GmbH, Woehrendamm 80, Großhansdorf 22927, Germany. E-mail: k.f.rabelalungenclinic.de

ABSTRACT This phase 2/3 randomised, parallel-group, placebo-controlled trial investigated oral corticosteroid (OCS)-sparing efficacy, safety and tolerability of nebulised budesonide (Bud) administered with a novel computer-controlled, compressor-driven inhalation system (AKITA) as add-on therapy to Global Initiative for Asthma step 5.

Patients (18-65 years) with OCS-dependent asthma were randomised (2:1:1:1) to receive 18-week, twicedaily, double-blind treatment with AKITA inhaled corticosteroid (AICS)-Bud $1 \mathrm{mg}$, AICS-Bud $0.5 \mathrm{mg}$, AICS-placebo or open-label Bud $1 \mathrm{mg}$ administered by conventional nebuliser (CN-Bud). OCS doses were tapered until week 14 .

199 patients started treatment. More AICS-Bud $1 \mathrm{mg}(80.0 \%)$ than placebo-treated $(62.5 \%)$ patients had daily OCS doses reduced $\geqslant 50 \%$, with clinical stability to week 18 (one-sided $p=0.02$; treatment difference: $17.5 \%$ (95\% CI $0.1-34.9 \%$ ), two-sided $\mathrm{p}=0.04$ ). Mean \pm sD forced expiratory volume in $1 \mathrm{~s}$ improved (from baseline to week 18) for AICS-Bud $1 \mathrm{mg}(239 \pm 460 \mathrm{~mL}, \mathrm{p}<0.001)$ and AICS-Bud $0.5 \mathrm{mg}(126 \pm 345 \mathrm{~mL}$, $\mathrm{p}=0.01)$ but not placebo $(93 \pm 419 \mathrm{~mL}, \mathrm{p}=0.36)$ or $\mathrm{CN}$-Bud $(137 \pm 459 \mathrm{~mL}, \mathrm{p}=0.18)$. Fewer AICS-Bud $1 \mathrm{mg}$ treated patients experienced asthma exacerbations (7.5\%) compared with placebo (17.5\%) or CN-Bud (22.5\%). All treatments were well tolerated.

Budesonide applied with AKITA allowed significant meaningful OCS reduction in OCS-dependent asthma patients while improving pulmonary function and maintaining exacerbation control.

@ERSpublications

Nebulised budesonide applied with AKITA allowed significant, meaningful OCS reduction in GINA step 5 asthma patients http://ow.ly/EpG7N 


\section{Introduction}

Asthma treatment guidelines (Global Initiative for Asthma (GINA)) recommend sequential treatments for asthma control, beginning with step 1 (rescue medication with short-acting $\beta_{2}$-agonists (SABA)) and step 2 (low-dose inhaled corticosteroids (ICS)), and escalating to step 5 (oral corticosteroids (OCS)) [1]. ICS treatment provides a favourable ratio of local antiasthma effects to systemic adverse effects. Despite poor pulmonary deposition, currently marketed ICS inhalation technologies (metered-dose inhalers (MDI), dry-powder inhalers (DPI) and conventional jet nebulisers $(\mathrm{CN})$ ) are highly efficacious for the majority of asthma patients. However, they provide suboptimal control in severe disease, with daily OCS required in $\sim 30 \%$ of adults with severe asthma [1-4]. Long-term OCS therapy is associated with significant adverse effects (e.g. weight gain, metabolic changes, osteoporosis and glaucoma) [5].

AKITA ICS (AICS)-budesonide (Bud) (Activaero GmbH, Gemuenden, Germany) is a novel medicinal product that combines the established safety and efficacy profile of nebulised ICS Bud [6, 7] with a patient-specific, computer-controlled, compressor-driven inhalation manoeuvre. The drug output and drug-deposition efficiency of traditional delivery devices are often affected by disease severity and/or a patient's individual breathing pattern. Furthermore, increasing the Bud dose in a traditional nebuliser would increase oropharyngeal deposition and side-effects [8].

The AKITA Inhalation System (fig. 1) uses a computer-controlled compressor that is programmed by an integrated circuit card (Smart Card) to control the entire inhalation manoeuvre. The device provides feedback to the user (e.g. remaining inhalations) and their physician (e.g. treatment compliance records). A patient-specific inhalation volume and slow $\left(200 \mathrm{~mL} \cdot \mathrm{s}^{-1}\right)$ positive-pressure air stream delivers nebulised Bud during the first part of the inhalation; the result is a favourable regional lung deposition profile (higher peripheral, and lower oropharyngeal and central deposition) and lower interpatient lung deposition variability, compared with $\mathrm{CN}$ [9-12].

Previous studies demonstrated the OCS-sparing efficacy of nebulised Bud and its potential advantages for treating asthma that is inadequately controlled using MDI/DPI [3, 4, 13-19]. However, systematic OCS tapering using nebulised Bud delivered with a patient-specific, controlled inhalation manoeuvre has never been investigated. Here, we report results of a phase 2/3 study investigating the OCS-sparing efficacy, safety and tolerability of AICS-Bud $1 \mathrm{mg}$ administered twice daily as add-on therapy to GINA step 5 treatments.

\section{Methods}

Patients

Patients ( $\geqslant 18$ and $\leqslant 65$ years) in this randomised, parallel, placebo-controlled trial had asthma ( $\geqslant 6$ months; allergic or nonallergic; American Thoracic Society (ATS) definition, pre-bronchodilator forced expiratory

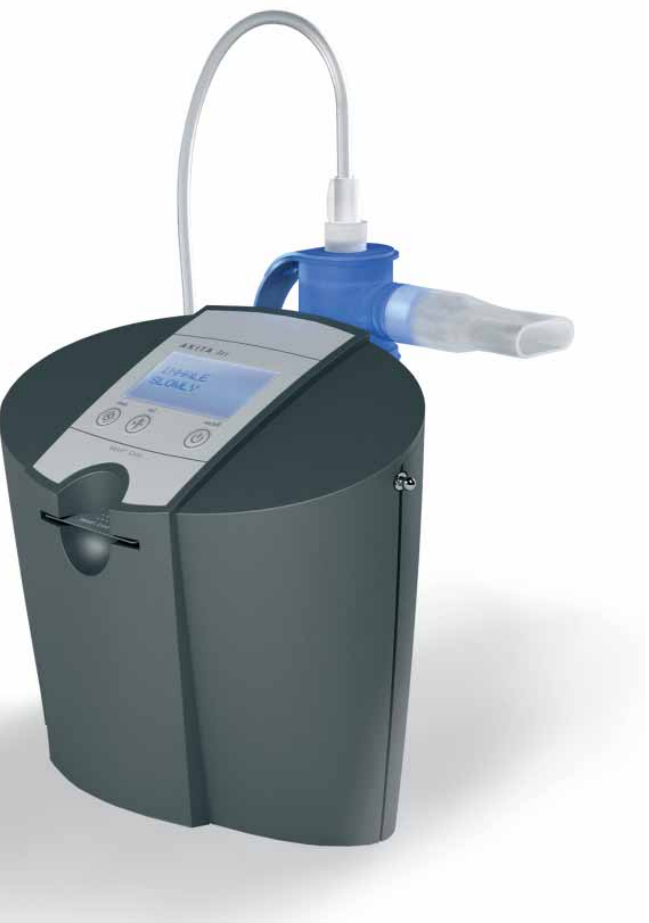

FIGURE 1 AKITA Inhalation System

(Activaero GmbH, Gemuenden, Germany). 
volume in $1 \mathrm{~s}(\mathrm{FEV} 1) \geqslant 40 \%$ and $\leqslant 79 \%$ predicted at screening or baseline; and documented $\geqslant 12 \%$ increase in FEV 1 15-30 min after SABA use (screening or previous 2 years)) that had been treated ( $\geqslant 3$ months) with high-dose ICS, OCS (mean daily dose $\geqslant 5$ and $<40 \mathrm{mg}$ ) and long-acting $\beta_{2}$-agonists.

Key exclusion criteria included: Bud allergy/reaction; other chronic lung diseases; hospitalisation for asthma, or use of anti-IgE, methotrexate, oral gold or intravenous $\gamma$-globulin (previous 3 months); upper respiratory tract infection or an emergency visit for asthma exacerbation (previous 4 weeks); use of other investigational asthma treatment (previous 30 days); current smoking; corticosteroid use for diseases other than asthma; women without acceptable birth control methods; and pregnant/nursing women.

\section{Study design}

This 24-week study (27 centres in Germany, Poland and Ukraine, from March 2010 to August 2011) included: a 4-week screening period to observe and adjust the OCS dose (according to GINA guidelines, the minimally effective dose should be used), and ensure sufficient adherence to therapy using diary cards; an 18-week treatment period (including a 14-week OCS-taper period); and a follow-up visit (week 20). Study visits occurred at 2-week intervals; baseline was the beginning of treatment.

Patients were randomised (2:1:1:1) to one of four treatment arms with $2 \mathrm{~mL}$ double-blind Bud 1 or $0.5 \mathrm{mg}$ (Infectopharm, Heppenheim, Germany) or placebo ( $0.9 \%$ saline; Pari GmbH, Starnberg, Germany) with the AKITA Inhalation System, or open-label BUD $1 \mathrm{mg}$ with a CN (CN-Bud; LC Spring, Pari GmbH); treatments were administered twice daily. Randomisation was stratified by median OCS during screening (5-10 mg.day ${ }^{-1} ;>10-40 \mathrm{mg} \cdot \mathrm{day}^{-1}$ ) and country. Parallel recruitment for all arms ensured any influences of seasonal environmental changes were equally distributed.

All patients received oral prednisone during the study; dose conversions from prednisolone or methylprednisolone used standard methods [20]. Patients recorded OCS and SABA doses and asthma medication in daily diaries, and continued their usual asthma medications (including ICS) during the study. Spirometry (ATS standards) was performed at each visit. FEV1 \% predicted was calculated using European Community for Coal and Steel reference standards [21].

Tapering of OCS doses occurred at 2-week intervals from weeks 2 to 14 (seven visits) if three criteria were met: FEV1 $\geqslant 80 \%$ of baseline value (pre-SABA); Asthma Control Questionnaire [22] symptom scores $\leqslant 120 \%$ or $<3$ points higher than baseline; and SABA $\leqslant 135 \%$ of baseline use. OCS doses were reduced by $5,2.5$ or $1 \mathrm{mg}$ if average daily doses were $>10,>5$ and $\leqslant 10$, or $\leqslant 5 \mathrm{mg}$, respectively. Increased need for OCS used the same stability criteria and reversed tapering steps.

Full physical examinations were performed at screening, baseline and study end, with abbreviated examinations at every visit.

Patients received written and verbal information about the purpose and nature of the study, and provided written informed consent before enrolment. This study was conducted in accordance with the principles of the Declaration of Helsinki. Independent ethics committees granted approval for each site. Patients were free to discontinue the study at any time.

\section{Outcome measures}

The primary end-point was the comparison of the percentages of patients whose median daily OCS dose during weeks $14-18$ was $\leqslant 50 \%$ of their median dose for the screening period and who remained clinically stable (no increase of more than two OCS-tapering steps according to stability criteria; no severe asthma exacerbations). Exacerbations were defined as a $\geqslant 25 \%$ decrease in FEV1 or an urgent/unscheduled visit for asthma symptoms, or $\geqslant 2$ consecutive days with $\geqslant 50 \%$ increases in SABA use or $\geqslant 25 \%$ decreases in morning peak expiratory flow (PEF).

Secondary efficacy end-points included assessments of OCS weaning, lung function, asthma exacerbations, hospitalisations, SABA use, quality of life (Mini Asthma Quality of Life Questionnaire scores [23]) and nocturnal awakenings. Exploratory assessments included comparing concomitant use of ICS, and statistical comparisons of changes in forced expiratory flow from $25 \%$ to $75 \%$ of vital capacity (FEF25-75\%), $\mathrm{FEV} 1$ and $\mathrm{FEV}_{1} \%$ predicted from baseline to week 18, and hospitalisation days as a fraction of study duration.

Serum Bud levels, urine cortisol/creatinine (UCC) ratios and serum cortisol were measured. Adverse events throughout the study were coded with Medical Dictionary for Regulatory Activities (version 13.0) terms.

\section{Statistics}

Statistical analyses (SAS, version 9.2; SAS Institute, Inc., Cary NC, USA) included 199 treated patients and used the last-observation-carried-forward convention. A sample size of 195 patients provided 81.2\% power to observe a significant difference between AICS-Bud $1 \mathrm{mg}$ and placebo for the primary end-point 
(one-sided $\alpha=0.025$ ) (ADDPLAN software, version 4.0; ADDPLAN GmbH, Cologne, Germany). The study used a two-stage group sequential adaptive design [24]. An unblinded, independent data monitoring committee reviewed sample size during an interim analysis after 65 patients were enrolled; it was reported to be adequate. Categorical and numerical end-points were evaluated with Chi-squared tests and t-tests, respectively. Change in FEV1 (from baseline to week 18) was evaluated using one-sample t-tests if the Kolmogorov-Smirnoff test did not suggest violation of Gaussian distribution; otherwise, Wilcoxon matched-pair tests were applied. Comparisons of AICS-Bud $1 \mathrm{mg}$ with AICS-Bud $0.5 \mathrm{mg}$ or CN-Bud were unpowered, descriptive comparisons. Randomisation (performed by AptivSolutions, Cologne, Germany) used a randomly permuted-block method.

\section{Results}

Of 220 screened patients, 200 were randomised, and 199 were treated and included in study analyses; $87.5 \%$ of patients (175 out of 200) completed the study (fig. 2). Dosing compliance (Smart Card adherence records) of $\geqslant 80 \%$ was reported for $71.3 \%$ of the AICS-Bud $1 \mathrm{mg}$ (57 out of 80 ), $62.5 \%$ of the AICS-placebo (25 out of 40 ) and $84.6 \%$ of the AICS-Bud $0.5 \mathrm{mg}$ (33 out of 39) arms. Mean compliance (counting used/unused vials) was $>85 \%$ of expected for time on study for each treatment arm.

Demographic and baseline data were comparable between arms (table 1). According to GINA guidelines [1], high-dose Bud is $>800 \mu \mathrm{g}$ per day. For 3 months before the study, patients used concomitant ICS via an inhaler at a mean \pm SD dose of $1378.1 \pm 965.5 \mu \mathrm{g}$ Bud equivalent per day, consistent with severe, uncontrolled asthma.

\section{Efficacy}

Significantly more AICS-Bud $1 \mathrm{mg}$ treated $(80.0 \%, 64$ out of 80$)$ than placebo-treated $(62.5 \%, 25$ out of 40) patients reduced their median daily OCS dose by $\geqslant 50 \%$ from baseline and remained clinically stable to week 18 ( $\mathrm{p}=0.02$, one-sided; table 2 and fig. 3a). The treatment difference was $17.5 \%$ (95\% CI $0.1-34.9 \%$, two-sided $\mathrm{p}=0.04)$.

Mean \pm SD reduction in OCS dose was $7.1 \pm 6.0 \mathrm{mg}$ for AICS-Bud $1 \mathrm{mg}$ and $3.6 \pm 6.7 \mathrm{mg}$ for placebo (treatment difference: $-3.5 \mathrm{mg}$, 95\% CI $-5.9--1.1 \mathrm{mg} ; \mathrm{p}=0.005)$. Full OCS weaning occurred for a numerically larger percentage of AICS-Bud $1 \mathrm{mg}(56.3 \%)$ than placebo-treated patients $(42.5 \%)(\mathrm{p}=0.155)$; note that full weaning was possible only with baseline OCS doses $<15 \mathrm{mg}$ per day due to the limited number of tapering steps. The incidence of exacerbation was numerically lower in the AICS-Bud $1 \mathrm{mg}$ arm versus placebo ( $7.5 \%$ versus $17.5 \%, \mathrm{p}=0.10$; fig. $3 \mathrm{~b}$ ); however, significantly fewer days were spent in hospital in relation to total study duration in the AICS-Bud $1 \mathrm{mg}$ arm (5 of 13152 versus 49 of 6328 days, $\mathrm{p}<0.001$; table 2). Mean \pm SD improvement from baseline FEV1 at week 18 was numerically larger for the AICS-Bud $1 \mathrm{mg}$ arm $(16.5 \pm 30.3 \%)$ than the placebo arm $(6.2 \pm 24.6 \%) \quad(\mathrm{p}=0.07)$, with a significant mean $\pm \mathrm{SD}$ improvement from baseline FEV1 observed for AICS-Bud $1 \mathrm{mg}(239 \pm 460 \mathrm{~mL} ; \mathrm{p}<0.001)$ but not for the

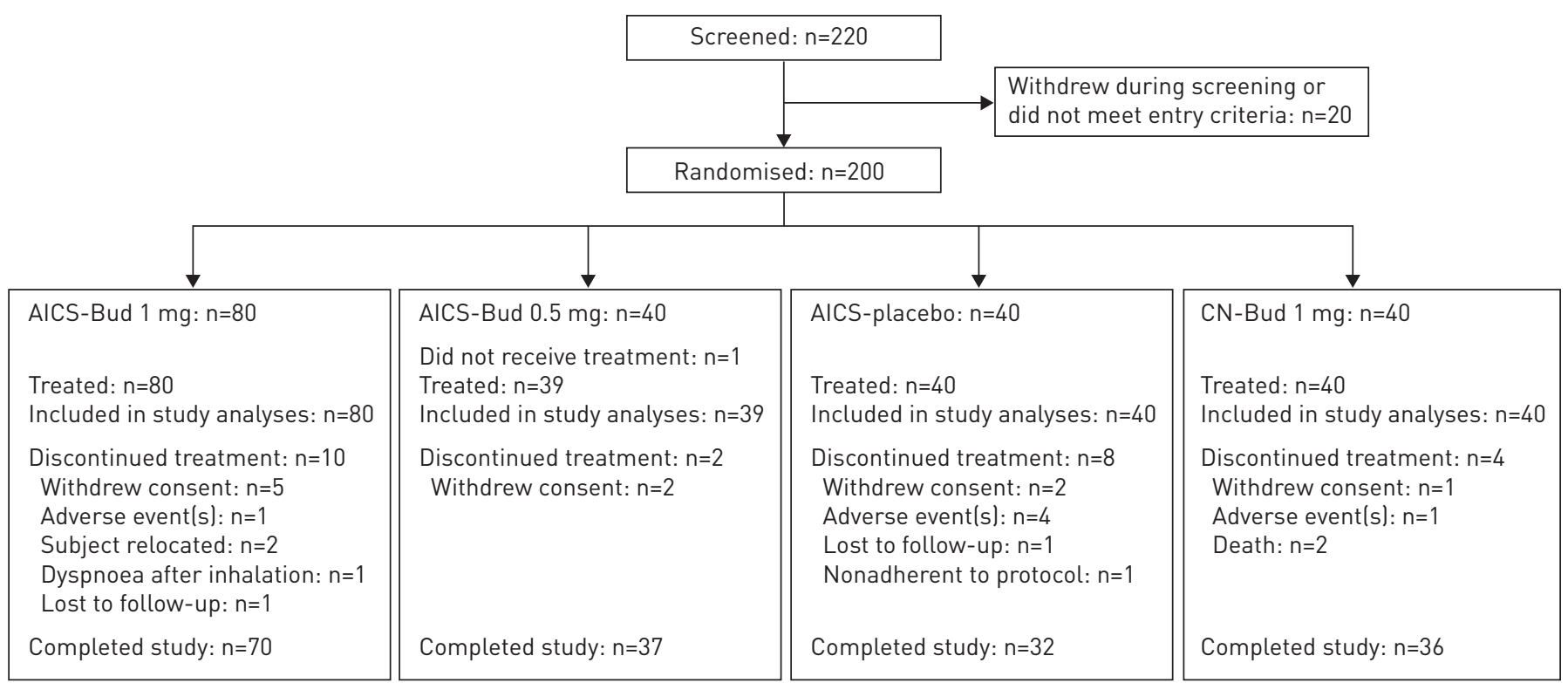




\begin{tabular}{|c|c|c|c|c|c|}
\hline & $\begin{array}{l}\text { AICS-Bud } \\
1 \mathrm{mg}\end{array}$ & $\begin{array}{l}\text { AICS-Bud } \\
0.5 \mathrm{mg}\end{array}$ & AICS-placebo & CN-Bud & All patients \\
\hline Patients n & 80 & 39 & 40 & 40 & 199 \\
\hline Age years & $52.0 \pm 8.8$ & $51.6 \pm 10.0$ & $52.3 \pm 9.2$ & $49.7 \pm 10.6$ & $51.5 \pm 9.5$ \\
\hline Males & $24(30.0)$ & $14(35.9)$ & $16(40.0)$ & $16(40.0)$ & $70(35.2)$ \\
\hline Females & $56(70.0)$ & $25(64.1)$ & $24(60.0)$ & $24(60.0)$ & $129(64.8)$ \\
\hline BMI $\mathrm{kg} \cdot \mathrm{m}^{-2}$ & $28.2 \pm 5.5$ & $28.7 \pm 5.4$ & $27.8 \pm 5.4$ & $27.7 \pm 3.8$ & $28.1 \pm 5.2$ \\
\hline Duration of asthma years & $19.2 \pm 12.1$ & $21.2 \pm 12.6$ & $19.9 \pm 11.7$ & $18.4 \pm 10.6$ & $19.6 \pm 11.8$ \\
\hline MiniAQLQ total score & $3.6 \pm 0.9$ & $3.7 \pm 1.0$ & $3.7 \pm 0.7$ & $3.6 \pm 1.0$ & $3.6 \pm 0.9$ \\
\hline ACQ total score & $3.2 \pm 0.8$ & $3.2 \pm 0.9$ & $3.0 \pm 0.7$ & $3.1 \pm 1.0$ & $3.1 \pm 0.8$ \\
\hline FEV $1 \%$ predicted & $59.0 \pm 11.8$ & $56.4 \pm 9.5$ & $57.0 \pm 11.2$ & $58.1 \pm 12.3$ & $57.9 \pm 11.3$ \\
\hline Morning PEF L-min ${ }^{-1}$ & $253 \pm 100^{\S}$ & $249 \pm 99^{f}$ & $269 \pm 101^{f}$ & $263 \pm 123^{f}$ & $257 \pm 104$ \\
\hline Evening PEF L-min ${ }^{-1}$ & $268 \pm 99 \$$ & $268 \pm 98^{f}$ & $286 \pm 101^{f}$ & $272 \pm 124^{f}$ & $272 \pm 104$ \\
\hline FEF $25-75 \%$ L.s ${ }^{-1}$ & $1.11 \pm 0.56^{\# \#}$ & $1.04 \pm 0.43^{\text {กๆ }}$ & $1.00 \pm 0.51^{f}$ & 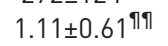 & ND \\
\hline \multicolumn{6}{|l|}{ oCs use ${ }^{\#}$} \\
\hline Baseline dose mg per day & $10.0 \pm 7.1$ & $10.6 \pm 9.0$ & $10.4 \pm 8.2$ & $10.1 \pm 6.2$ & $10.2 \pm 7.5$ \\
\hline Use of 5-10 mg per day & $63(79)$ & $32(82)$ & 31 (78) & $30(75)$ & $156(78)$ \\
\hline SABA puffs per day & $4.6 \pm 3.7^{++}$ & $4.0 \pm 3.6^{\S \S}$ & $4.3 \pm 3.6^{f f}$ & $4.3 \pm 3.1^{f}$ & $4.4 \pm 3.5^{\# \# \#}$ \\
\hline \multicolumn{6}{|l|}{$\begin{array}{l}\text { Most common inhaled } \\
\text { asthma medications }\end{array}$} \\
\hline Salbutamol & 74 (92.5) & 36 (92.3) & 35 (87.5) & 37 (92.5) & 182 (91.5) \\
\hline Fluticasone/salmeterol & $29(36.3)$ & $12(30.8)$ & $16(40.0)$ & $17(42.5)$ & $74(37.2)$ \\
\hline Formoterol & $20(25.0)$ & 15 (38.5) & 9 (22.5) & $10(25.0)$ & $54(27.1)$ \\
\hline Fluticasone & $18(22.5)$ & $9(23.1)$ & 13 (32.5) & $8(20.0)$ & $48(24.1)$ \\
\hline Salmeterol & $18(22.5)$ & $6(15.4)$ & $10(25.0)$ & $10(25.0)$ & $44(22.1)$ \\
\hline Bud/formoterol fumarate & $13(16.3)$ & $5(12.8)$ & $4(10.0)$ & $3(7.5)$ & $25(12.6)$ \\
\hline Bud & $12(15.0)$ & $4(10.3)$ & $3(7.5)$ & $6(15.0)$ & $25(12.6)$ \\
\hline Beclometasone & $8(10.0)$ & $8(20.5)$ & $3(7.5)$ & $2(5.0)$ & $21(10.6)$ \\
\hline Fenoterol & $7(8.8)$ & $5(12.8)$ & $3(7.5)$ & $3(7.5)$ & $18(9.0)$ \\
\hline Tiotropium & $5(6.3)$ & $6(15.4)$ & $3(7.5)$ & $2(5.0)$ & $16(8.0)$ \\
\hline Ex-smokers $^{+}$ & $7(8.8)$ & $4(10.3)$ & $3(7.5)$ & $9(22.5)$ & $23(11.6)$ \\
\hline Exposure pack-years & $5.3 \pm 3.0$ & $5.5 \pm 2.6$ & $8.0 \pm 1.0$ & $5.2 \pm 3.1$ & $5.7 \pm 2.8$ \\
\hline
\end{tabular}

Data are presented as mean \pm SD or $n(\%)$ unless otherwise stated. AICS: AKITA inhaled corticosteroid; Bud: budesonide; CN: conventional jet nebuliser; BMI: body mass index; AQLQ: Asthma Quality of Life Questionnaire; ACQ: Asthma Control Questionnaire; FEV1: forced expiratory volume in $1 \mathrm{~s}$; PEF: peak expiratory flow; FEF25-75\%: forced expiratory flow from $25 \%$ to $75 \%$ of vital capacity; OCS: oral corticosteroids; SABA: short-acting $\beta_{2}$-agonists; ND: not determined. \# : all patients were receiving OCS; prednisone was specified by the protocol for use during the study; " : study eligibility specified that all patients were receiving long-acting $\beta_{2}$-agonists; ${ }^{+}$: study eligibility specified patients were nonsmokers or

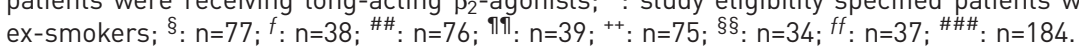

placebo arm $(93 \pm 419 \mathrm{~mL} ; \mathrm{p}=0.36)$ (fig. 3c). The larger improvements observed for AICS-Bud $1 \mathrm{mg}$ relative to placebo were supported by results of additional secondary end-points (table 2), including reduced SABA use (AICS-Bud $1 \mathrm{mg}$ versus placebo treatment difference: -1.2 puffs per day; $\mathrm{p}=0.008$ ) and fewer patients hospitalised (AICS-Bud $1 \mathrm{mg}$ : 1.3\%; placebo: 10\%; $\mathrm{p}=0.01$ ). Mean \pm SD change in $\mathrm{FEF} 25-75 \%$ from baseline to week 18 was $+0.20 \pm 0.60 \mathrm{~L} \cdot \mathrm{s}^{-1}$ for AICS-Bud $1 \mathrm{mg}$ and $0.00 \pm 0.40 \mathrm{~L} \cdot \mathrm{s}^{-1}$ for placebo $(\mathrm{p}=0.03)$.

Efficacy responses for AICS-Bud $0.5 \mathrm{mg}$ were generally comparable to those for AICS-Bud $1 \mathrm{mg}$, except lung function improvements were somewhat lower (table 2 and fig. 3c). Most efficacy responses for CN-Bud $1 \mathrm{mg}$ were comparable to those observed for AICS-Bud 1 or $0.5 \mathrm{mg}$, except more CN-Bud $1 \mathrm{mg}$-treated than AICS-Bud 1 mg-treated patients experienced asthma exacerbations ( $22.5 \%$ versus $7.5 \%$; $\mathrm{p}=0.02)$, mean time to first exacerbation was shorter (50.1 versus 96.5 days) and a larger percentage of patients experienced asthma instability (25.0\% versus $15.0 \%$ ) (table 2 and fig. $3 \mathrm{~b}$ ), although the small number of patients experiencing asthma exacerbations/instability make it difficult to interpret these differences.

\section{Pharmacokinetics and clinical chemistry}

At each time-point, serum Bud levels after treatment with AICS-Bud $1 \mathrm{mg}$ were approximately two-fold higher than with AICS-Bud $0.5 \mathrm{mg}$ or CN-Bud $1 \mathrm{mg}$ (table 3). Bud did not accumulate significantly over the 18-week treatment period. Variability of post-inhalation serum Bud levels, estimated by coefficients of variation, was lower in the AICS-Bud arms (52-73\%) compared with CN-Bud (69-85\%). 
TABLE 2 Efficacy results

AICS-Bud $1 \mathrm{mg} \quad$ AICS-Bud $0.5 \mathrm{mg} \quad$ AICS-Placebo $\quad$ CN-Bud $1 \mathrm{mg}$

80

31

25

30

\section{Primary end-point}

Reduction of OCS $\geqslant 50 \%$ from baseline with clinical stability to 18 weeks ${ }^{\#}$

Secondary end-points

OCS

Fully weaned off OCS at 18 weeks

Reduction of daily OCS from baseline to 18 weeks $\%$

Absolute reduction of daily OCS from baseline to

18 weeks $\mathrm{mg}$

Lung function

Change in FEV1 from baseline to 18 weeks \%

Absolute change in $\mathrm{FEV}_{1}$ from baseline to

18 weeks $\%$ pred

Absolute change in morning PEF from baseline to

18 weeks L. $\mathrm{min}^{-1}$

Absolute change in evening PEF from baseline to 18 weeks L. min $^{-1}$

Absolute change in $\mathrm{FEF}_{25}-75 \%$ from baseline to

18 weeks ${ }^{\mathbb{1}} \mathrm{L} \cdot \mathrm{min}^{-1}$

Exacerbations and instability

$\geqslant 1$ asthma exacerbation

Time to first asthma exacerbation days

$\geqslant 1$ severe asthma exacerbation

Asthma instability ${ }^{+}$

Absolute change in SABA dose from baseline to

18 weeks puffs per day

Bud-equivalent inhaled corticosteroid dose ${ }^{\S} \mu$ g per day

Patients hospitalised for asthma

Total time hospitalised/total study duration ${ }^{f}$ days

ACQ question 1: nocturnal awakenings $\#$

Improvement

No change

Deterioration

No data

Quality of life

Change in MiniAQLQ score from baseline to 18 weeks

$64(80.0)^{*}$

$31(79.5)$

$25(62.5)$

$30(75.0)$
$45(56.3)$

$74.8 \pm 48.9 *(n=77)$

$7.1 \pm 6.0^{* *}(n=77)$

$16.5 \pm 30.3(n=76)$

$8.8 \pm 16.7(n=76)$

$25.3 \pm 65.6(n=74)$

$22.6 \pm 60.4(n=74)$

$+0.20 \pm 0.60 *(n=76)$

$6(7.5)$

$96.5 \pm 51.2(n=6)$

$1(1.3)$

$12(15.0)$

$-1.5 \pm 2.5^{* *}(\mathrm{n}=70)$

$1349.6 \pm 1189.3$

$1(1.3)^{*}$

$5 / 13152^{* * *}$

19 (23.8)

11 (13.8)

$4(5.0)$

$0.83 \pm 1.16 *(n=73)$
46 (57.5)

20 (51.3)

$77.5 \pm 31.6(\mathrm{n}=38)$

$7.6 \pm 6.2(n=38)$

17 (42.5)

$51.7 \pm 62.9(\mathrm{n}=40)$

$3.6 \pm 6.7(n=40)$

$18(45.0)$

$70.6 \pm 59.6(n=39)$

$6.8 \pm 5.7(n=39)$

\section{$9.5 \pm 20.7(n=39)$}

$5.4 \pm 11.5(n=39)$

$6.2 \pm 24.6(n=39)$

$3.0 \pm 13.5(n=39)$

$8.6 \pm 27.8(n=39)$

$4.0 \pm 14.3(n=39)$

\section{$17.8 \pm 39.0(n=38)$}

$14.9 \pm 47.6(n=37)$

$28.2 \pm 56.7(n=37)$

$12.1 \pm 44.6(n=37)$

$7.8 \pm 56.9(n=37)$

$31.0 \pm 57.8(n=37)$

$+0.08 \pm 0.44(n=39)$

$0.00 \pm 0.40(n=38)$

$+0.05 \pm 0.71(n=39)$
3 (7.7)

$87.7 \pm 47.0(n=3)$

$1(2.6)$

5 (12.8)

$-1.4 \pm 2.5(n=37)$

$1253.8 \pm 743.5$

$1(2.6)$

$5 / 6568$

21 (53.8)

15 (38.5)

$3(7.7)$

$0(0)$
7 (17.5)

$47.9 \pm 34.7(n=7)$

3 (7.5)

$12(30.0)$

$-0.4 \pm 1.8(n=33)$

$1627.0 \pm 872.0$

$4^{++}(10)$

49/6328

17 (42.5)

$14(35.0)$

7 (17.5)

2 (5.0)
9 (22.5)

$50.1 \pm 35.3(n=9)$

1 (2.5)

$10(25.0)$

$-1.6 \pm 2.9(n=35)$

$1329.3 \pm 717.5$

1 (2.5)

$5 / 6660$

23 (57.5)

$12(30.0)$

$4(10.0)$

1 (2.5)

Data are presented as $\mathrm{n}(\%)$ unless otherwise stated. AICS: AKITA inhaled corticosteroid; Bud: budesonide; CN: conventional jet nebuliser; OCS: oral corticosteroids; FEV1: forced expiratory volume in $1 \mathrm{~s}$; PEF: peak expiratory flow; FEF25-75\%: forced expiratory flow from $25 \%$ to $75 \%$ of vital capacity; SABA: short-acting $\beta_{2}$-agonists; ACQ: Asthma Control Questionnaire; AQLQ: Asthma Quality of Life Questionnaire. \#: patients with severe exacerbations (requiring hospitalisation/emergency intervention) and mild-moderate exacerbations requiring OCS increases of more than two reverse-tapering steps were considered not to have reached the primary end-point. ": exploratory analysis; missing data imputed using last observation carried forward; compared with Wilcoxon-Mann-Whitney test. ${ }^{+}$: increase in OCS dose required; increases were made according to the stability criteria and using the reverse of the OCS-tapering steps. ${ }^{\S}$ : calculated using the inhaled corticosteroid conversion tables found in the British Guideline on the Management of Asthma [25]; concomitant use of inhaled corticosteroid is presented; use remained unchanged during the study. ${ }^{f}$ : compared with Fisher's exact probability. ${ }^{\# \#}$ : improvement or deterioration defined as change of $\geqslant 1$ point from baseline to week 18. กाก: minimal clinically important difference, 0.5 points. ${ }^{++}$: one patient also had pseudomonal lung infection. *: p<0.05 comparing AICS-Bud 1 mg with AICS-placebo; ${ }^{* *}$ : $p<0.01$ comparing AICS-Bud 1 mg with AICS-placebo; ${ }^{* * *} p<0.001$ comparing AICS-Bud 1 mg with AICS-placebo.

Low morning mean serum cortisol levels and UCC ratios indicated that the hypothalamic-pituitaryadrenal (HPA) axis was suppressed in a substantial portion of patients at baseline (table 3). Endogenous cortisol levels did not change significantly throughout the study in any arm. UCC ratios remained almost unchanged in the three Bud arms and increased slightly in the placebo arm, probably because placebo-treated patients did not inhale additional Bud and substantially reduced their OCS doses.

\section{Safety}

Treatment-emergent adverse events reported for $\geqslant 10 \%$ of patients in any arm included nasopharyngitis (AICS-Bud $1 \mathrm{mg}$ : 17.5\%; AICS-Bud $0.5 \mathrm{mg}$ : 17.9\%; placebo: 17.5\%; CN-Bud: 12.5\%), asthma (7.5\%, 10.3\%, $22.5 \%$ and $22.5 \%$, respectively) and respiratory tract infection (11.3\%, 7.7\%, 5.0\% and $12.5 \%)$. Dysphonia was most commonly reported treatment-related event (investigator judgment) (AICS-Bud $1 \mathrm{mg}$ : four out 

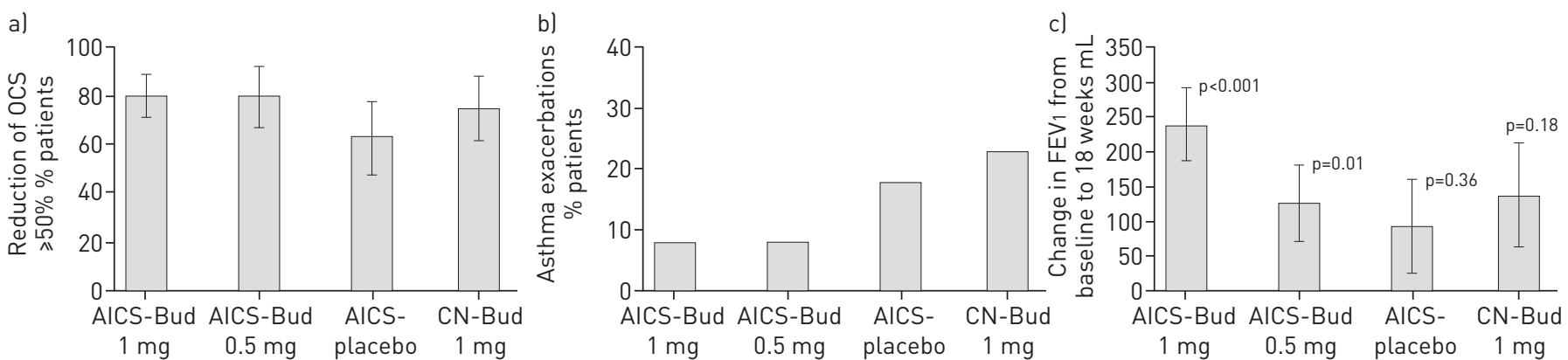

FIGURE 3 Efficacy parameters. a) Percentage of patients with a $\geqslant 50 \%$ reduction from baseline oral corticosteroids (OCS) (prednisone) dose, accompanied by clinical stability to week 18 (end of treatment). Whiskers represent $95 \%$ confidence intervals. b) Percentage of patients with asthma exacerbation(s) during the 18-week treatment period. c) Mean absolute change from baseline forced expiratory volume in $1 \mathrm{~s}$ (FEV1) at week 18 (end of treatment). Whiskers represent the standard error. p-values are from one-sample t-tests comparing baseline and week 18 for AKITA inhaled corticosteroid (AICS)-budesonide (Bud) 1 mg and from Wilcoxon matched-pairs tests for the other three arms. CN: conventional jet nebuliser.

of 80 patients; AICS-Bud $0.5 \mathrm{mg}$ : one out of 39 patients; placebo: one out of 40 patients). Overall, dysphonia occurred primarily in patients (six out of seven patients) using fluticasone as the concomitant ICS. The incidence of other potential local adverse reactions to ICS was low: events corresponding to fungal infections of the mouth/oropharynx were reported in three Bud-treated and two placebo-treated patients, and dyspnoea and cough were each reported in two Bud-treated patients. Asthma was the only severe/life-threatening event experienced by more than one patient in any arm (AICS-Bud $1 \mathrm{mg}$ : one out of 80 patients; AICS-Bud 0.5 mg: two out of 39 patients; placebo: five out of 40 patients; CN-Bud: one out of 40 patients); none of the severe/life-threatening events were considered treatment-related.

Two patients died during the study (CN-Bud arm; subarachnoid haemorrhage and pulmonary haemorrhage); these deaths were not treatment related (investigator judgment).

Adverse events leading to study discontinuation included asthma exacerbation (AICS-Bud $1 \mathrm{mg}$ : one out of 80 patients; placebo: three out of 40 patients; $\mathrm{CN}$-Bud: one out of 40 patients) and oropharyngeal candidiasis (placebo: one out of 40 patients). Adverse events leading to study treatment discontinuation included oral candidiasis (AICS-Bud $1 \mathrm{mg}$ : one out of 80 patients), dysphonia (AICS-Bud $0.5 \mathrm{mg}$ : one out of 29 patients), and dysphonia and oropharyngeal pain (placebo: one out of 40 patients); these three patients discontinued the study due to withdrawal of consent.

\section{Discussion}

This placebo-controlled study evaluated AICS-Bud $1 \mathrm{mg}$ twice daily as an add-on therapy to GINA step 5 treatment recommendations. AICS-Bud $1 \mathrm{mg}$ enabled patients with severe, OCS-dependent asthma to significantly reduce their OCS dose, maintain control over asthma exacerbations and improve lung

TABLE 3 Pharmacokinetics and clinical chemistry

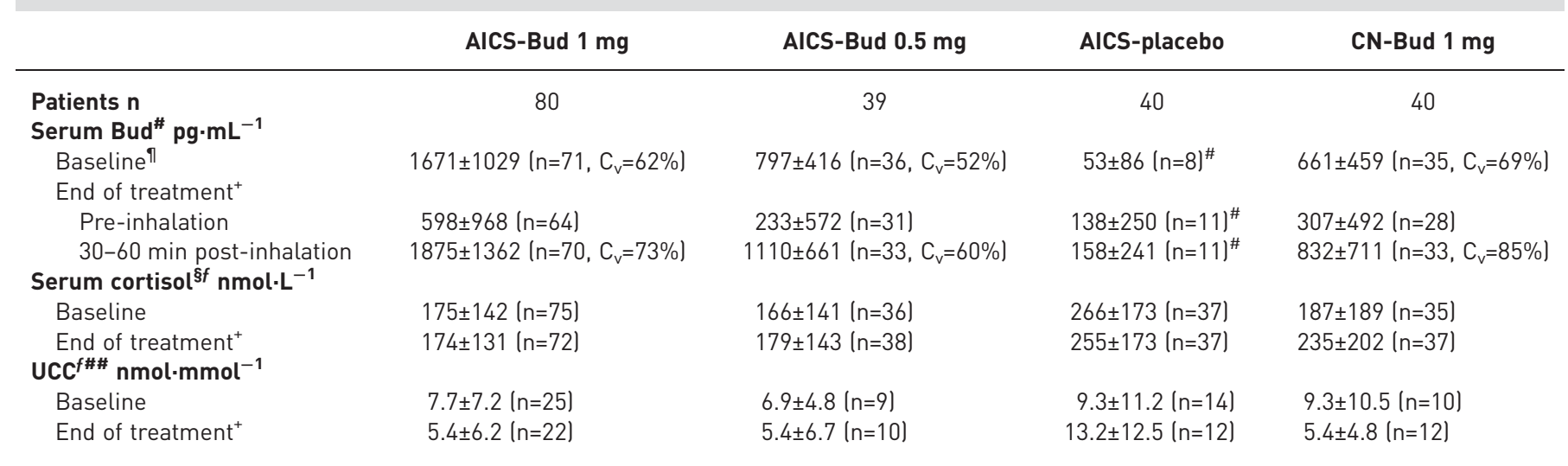

Data are presented as mean \pm SD unless otherwise stated. AICS: AKITA inhaled corticosteroid; Bud: budesonide; CN: conventional jet nebuliser; $\mathrm{C}_{\mathrm{v}}$ : coefficient of variation; UCC: urine cortisol/creatinine. \# : data for patients with levels below the lower level of quantification are not included in the table; ${ }^{\text {ๆ }}$ : 30-60 min post-inhalation; ${ }^{+}$: week $18 ;{ }^{\S}$ : serum cortisol reference range $171-535 \mathrm{nmol}^{-\mathrm{L}^{-1}}{ }^{\dagger}$ : mean values from samples obtained at 09:00 $\mathrm{h} ;{ }^{\# \#}$ : measured only for patients in Germany and Poland. 
function, compared with placebo. While the incidence of exacerbation was numerically lower in the AICS-Bud $1 \mathrm{mg}$ arm versus placebo, significantly fewer days were spent in hospital in relation to total study duration. The study was not powered to evaluate severe exacerbations nor was the duration sufficient to assess them. As significant adverse effects are associated with OCS, considerable health improvements can be expected from reducing/eliminating OCS use while maintaining asthma symptom control.

Some OCS dose reduction was possible in the control (placebo) and comparator (AICS-Bud $0.5 \mathrm{mg}$ and $\mathrm{CN}$-Bud $1 \mathrm{mg}$ ) arms. However, improvements in lung function were numerically lower for AICS-Bud $0.5 \mathrm{mg}$ compared with AICS-Bud $1 \mathrm{mg}$, and the incidence of asthma exacerbation was higher for placebo and CN-Bud $1 \mathrm{mg}$. Small airways in the periphery of the lung contribute significantly to airflow limitation in asthma [26]; thus, the lower incidence of asthma exacerbation in the AICS-Bud arms may be explained by higher peripheral lung drug deposition. This interpretation is supported by the improved FEF25-75\% and the slightly higher systemic Bud levels observed in the AICS-Bud $1 \mathrm{mg}$ arm, compared with either placebo or CN-Bud $1 \mathrm{mg}$.

The overall incidence of adverse events was low, probably because patients continued their usual asthma treatments that were consistent with GINA step 5 recommendations. Endogenous cortisol levels revealed that the HPA axis remained relatively unaffected by AICS-Bud treatment; levels were measured only for a subset of patients and dropped slightly from baseline to the end of treatment in the three arms receiving Bud. A long-term study may be needed to confirm the effects of AICS-Bud on key parameters such as HPA axis integrity, osteoporosis and infections.

Because the contribution of swallowed drug to systemic levels is negligible during the first 30-60 min following inhalation, drug serum concentration during this time provides an index of lung deposition [27]. Together with the exacerbation incidences, the serum Bud data suggest that total and peripheral lung deposition with AICS-Bud may be more efficient and less variable compared with the conventional nebuliser. The clinical implications are important for patients with severe asthma: reliance on a systemic steroid to control exacerbations may lessen when locally acting steroids reliably reach affected airways. Since study patients were dependent on OCS despite receiving high-dose ICS via an inhaler (MDI/DPI), we can infer that for some severe asthma patients, inhalers may not be fully effective ICS delivery devices.

Although the study was only powered to demonstrate superiority of AICS-Bud $1 \mathrm{mg}$ over placebo, the dose-response relationship with FEV 1 and PEF observed between AICS-Bud $1 \mathrm{mg}$ and $0.5 \mathrm{mg}$ tended to favour AICS-Bud $1 \mathrm{mg}$. This observation is consistent with a meta-analysis of results from trials enrolling adult patients with suboptimally controlled severe asthma treated with OCS; FEV1 and morning PEF both improved on $1600 \mu \mathrm{g}$ per day inhaled Bud compared with $200 \mu \mathrm{g}$ per day [28].

In some previous OCS-sparing asthma studies of patients using high-dose ICS, patients discontinued all ICS upon enrolment, i.e. the study drug/placebo replaced patient's ICS therapy during OCS tapering $[13,29]$. We believe the design employed during our trial (AICS-Bud as add-on therapy to replace OCS but not other ICS) represents a scenario that will be easier to implement outside the context of a clinical trial and that is more in line with GINA step 5 recommendations. Furthermore, the results of this study suggest that the dose-response relationship for inhaled ICS in GINA step 5 asthma may not be as flat as generally believed [29]. It has to be considered, however, that existing ICS therapy was not standardised in this study and that Bud-equivalent doses show a high standard deviation, possibly indicating that some patients were not on high-dose ICS, as warranted by GINA guidelines.

For a study design using stepwise, methodical OCS reductions, the expected outcome is stability of disease indicators and not necessarily improvement. Hence, the improvement of key secondary end-points (e.g. asthma exacerbations/instability, hospitalisations, lung function and $\beta$-agonist use) for AICS-Bud $1 \mathrm{mg}$-treated patients compared with either placebo or $\mathrm{CN}$-Bud $1 \mathrm{mg}$ was a very positive but somewhat surprising observation. This design (stepwise, methodical substitution of an effective (and potentially unsafe) therapy with one having a more favourable risk/benefit profile) has precedents in the asthma literature $[30,31]$.

The high placebo response rate suggests that some study patients may have been overtreated with OCS upon enrolment or that adherence to OCS therapy was not sufficient prior to study entry, although investigators made sure that adherence was sufficiently high during the run-in phase using diary cards. During the treatment phase, high compliance rates were observed in this study; $62.5 \%$ of placebo-treated and $71.3 \%$ of AICS-Bud $1 \mathrm{mg}$-treated patients received $\geqslant 80 \%$ of expected doses, with mean compliance $>85 \%$ in both arms, which is higher than reported in an observational study [32]. A longer run-in period with an OCS dose reduction protocol would ensure that patients were using the minimally effective OCS dose at study start, although risk of a higher drop-out rate due to exacerbations would increase. Due to very low prevalence, recruiting patients with severe, OCS-dependent asthma for this study was challenging. 
Hence, it may not be feasible to run a trial that is powered for significance and that features a prolonged run-in to reduce OCS to a minimally effective dose.

In conclusion, this study demonstrated that treatment with AICS-Bud $1 \mathrm{mg}$ twice daily enables GINA step 5 asthma patients to reduce daily OCS without compromising asthma symptom and exacerbation control or reducing lung function. AICS-Bud $1 \mathrm{mg}$ was superior to treatment with placebo and some secondary end-points indicated that it may also have been superior to treatment with Bud-CN, although the study was neither designed nor powered to test this. AICS-Bud, a novel nebuliser-based inhalation product, provides increased therapeutic benefit as a result of a slow, controlled, patient-tailored inhalation manoeuvre. Since long-term use of OCS is associated with significant adverse effects, GINA step 5 asthma patients would benefit from this alternative therapy.

\section{Acknowledgements}

Data analysis was conducted by Andrea Kreter (ClinResearch, Cologne, Germany) under the sponsorship of Activaero $\mathrm{GmbH}$. Medical writing assistance was provided by Kathleen Simis (freelance medical writer) and Kate Loughney (KML Medical Communications, Seattle, WA, USA) under the sponsorship of Activaero.

\section{References}

1 Global Strategy for Asthma Management and Prevention 2008 (update). www.ginasthma.org. Date last accessed: July 9, 2014.

2 Jarjour NN, Erzurum SC, Bleecker ER, et al. Severe asthma: lessons learned from the National Heart, Lung, and Blood Institute Severe Asthma Research Program. Am J Respir Crit Care Med 2012; 185: 356-362.

3 Connolly KC, Peake MD, Halpin DMG, et al. Challenging current asthma treatment guidelines. Dis Manag Health Outcomes 2000; 7: 217-225.

4 Gawchik SM. Successful treatment of previously uncontrolled adult asthma with budesonide inhalation suspension: five-year case histories. Ann Pharmacother 2007; 41: 1728-1733.

5 Walsh LJ, Wong CA, Oborne J, et al. Adverse effects of oral corticosteroids in relation to dose in patients with lung disease. Thorax 2001; 56: 279-284.

6 Cruz-Rivera M, Lyzell E, Fitzpatrick S. Low frequency of adverse events reported through postmarketing surveillance for Pulmicortrespules ${ }^{\mathrm{TM}}$ (budesonide inhalation suspension) in the US adult population (abstract 895). J Allergy Clin Immunol 2002; 109: Suppl. 1, S292-S293.

7 Lyzell E, Cruz-Rivera M, Fitzpatrick S. Safety of Pulmicortrespules ${ }^{\mathrm{TM}}$ (budesonide inhalation suspension) in geriatric patients: postmarketing surveillance and clinical study data (abstract 894). J Allergy Clin Immunol 2002; 109: Suppl. 1, S292.

8 Bisgaard H, Nikander K, Munch E. Comparative study of budesonide as a nebulized suspension vs pressurized metered-dose inhaler in adult asthmatics. Respir Med 1998; 92: 44-49.

9 Brand P, Friemel I, Meyer T, et al. Total deposition of therapeutic particles during spontaneous and controlled inhalations. J Pharm Sci 2000; 89: 724-731.

10 Brand P, Beckmann H, Maas Enriquez M, et al. Peripheral deposition of $\alpha_{1}$-protease inhibitor using commercial inhalation devices. Eur Respir J 2003; 22: 263-267.

11 Fischer A, Stegemann J, Scheuch G, et al. Novel devices for individualized controlled inhalation can optimize aerosol therapy in efficacy, patient care and power of clinical trials. Eur J Med Res 2009; 14: Suppl. 4, 71-77.

12 Scheuch G, Brand P, Meyer T, et al. Regional drug targeting within the lungs by controlled inhalation with the AKITA-inhalation system. Respir Drug Del VIII 2002; 2: 471-474.

13 Crowe M, Gay AL, Keelan P. Prednisolone sparing effect of high dose budesonide aerosol in the management of chronic systemic steroid dependent asthmatics. Ir Med J 1986; 79: 39-41.

14 Higenbottam TW, Britton J, Lawrence D, et al. Comparison of nebulised budesonide and prednisolone in severe asthma exacerbation in adults. BioDrugs 2000; 14: 247-254.

15 Otulana BA, Varma N, Bullock A, et al. High dose nebulized steroid in the treatment of chronic steroid-dependent asthma. Respir Med 1992; 86: 105-108.

16 Ververeli K, Chipps B. Oral corticosteroid-sparing effects of inhaled corticosteroids in the treatment of persistent and acute asthma. Ann Allergy Asthma Immunol 2004; 92: 512-522.

17 Bisgaard H, Nikander K, Munch E. Comparative study of budesonide as a nebulized suspension vs pressurized metered-dose inhaler in adult asthmatics. Respir Med 1998; 92: 44-49.

18 Kamimura M, Izumi S, Hamamoto Y, et al. Superiority of nebulized corticosteroids over dry powder inhalers in certain patients with cough variant asthma or cough-predominant asthma. Allergol Int 2012; 61: 411-417.

19 Marcus P. Budesonide inhalation suspension in adults with poorly controlled asthma or chronic obstructive pulmonary disease. J Appl Res 2009; 9: 2-13.

20 GlobalRPh. Corticosteroid converter. www.globalrph.com/steroid.cgi Date last accessed: July 9, 2014. Date last updated: November 18, 2014.

21 Pellegrino R, Viegi G, Brusasco V, et al. Interpretative strategies for lung function tests. Eur Respir J 2005; 26 : 948-968.

22 Juniper EF, O'Byrne PM, Guyatt GH, et al. Development and validation of a questionnaire to measure asthma control. Eur Respir J 1999; 14: 902-907.

23 Juniper EF, Guyatt GH, Ferrie PJ, et al. Measuring quality of life in asthma. Am Rev Respir Dis 1993; 147: 832-838.

24 Lehmacher W, Wassmer G. Adaptive sample size calculations in group sequential trials. Biometrics 1999; 55: 1286-1290.

25 British Thoracic Society, Scottish Intercollegiate Guidelines Network. British guideline on the management of asthma: a national clinical guideline. www.brit-thoracic.org.uk/document-library/clinical-information/asthma/ btssign-guideline-on-the-management-of-asthma/ Date last accessed: July 9, 2014. Date last updated: January 2012. 
26 Burgel PR. The role of small airways in obstructive airway diseases. Eur Respir Rev 2011; 20: 23-33.

27 Newman SP. Can lung deposition data act as a surrogate for the clinical response to inhaled asthma drugs? $\mathrm{Br} \mathrm{J}$ Clin Pharmacol 2000; 49: 529-537.

28 Adams N, Bestall J, Jones PW. Budesonide at different doses for chronic asthma. Cochrane Database Syst Rev 2001; CD003271.

29 Ädelroth E, Rosenhall L, Glennow C. High dose inhaled budesonide in the treatment of severe steroid-dependent asthmatics. Allergy 1985; 40: 58-64.

30 Abuan T, Yeager M, Montgomery AB. Inhaled lidocaine for the treatment of asthma: lack of efficacy in two double-blind, randomized, placebo-controlled clinical studies. J Aerosol Med Pulm Drug Deliv 2010; 23: 381-388.

31 Walker S, Monteil M, Phelan K, et al. Anti-IgE for chronic asthma in adults and children. Cochrane Database Syst Rev 2006; CD003559.

32 Ismaila A, Corriveau D, Vaillancourt J, et al. Impact of adherence to treatment with fluticasone propionate/ salmeterol in asthma patients. Curr Med Res Opin 2014; 30: 1417-1425. 